\title{
Numerical modeling of the equilibrium shapes of a ferrofluid drop in an external magnetic field
}

\author{
O. Lavrova* ${ }^{* 1}$, G. Matthies ${ }^{* * 2}$, V. Polevikov ${ }^{* * 3}$, and L. Tobiska ${ }^{\dagger 1}$ \\ ${ }^{1}$ Institut für Analysis und Numerik, Otto-von-Guericke-Universität Magdeburg, PF4120, 39016 Magdeburg, Germany \\ ${ }^{2}$ Fakultät für Mathematik, Ruhr-Universität Bochum, Universitätsstraße 150, 44780 Bochum, Germany \\ ${ }^{3}$ Department of Computational Mathematics, Belarusian State University, F. Skaryna Ave. 4, 220050 Minsk, Belarus
}

\begin{abstract}
A numerical solution strategy for calculating equilibrium free surfaces of a magnetic fluid under the action of a magnetic field is proposed and applied to determine shapes of a linear magnetisable ferrofluid drop in a uniform magnetic field. Hysteresis phenomena for the drop deformation and the drop shapes with ends, close to conical, were observed numerically.
\end{abstract}

(C) 2004 WILEY-VCH Verlag GmbH \& Co. KGaA, Weinheim

\section{Introduction}

The main objective of the work is to develop a numerical solution strategy for calculating equilibrium shapes of a ferrofluid drop subjected to a uniform applied magnetic field. The free boundary value problem is governed by a coupled system of Maxwell's equations for the magnetic field distribution and Young-Laplace equation for the free surface shape. Maxwell's equations are formulated in the domain with an a priori unknown fluid-air interface. Both the drop shape and the magnetic field have to be found simultaneously. We split the nonlinear system of equations into subproblems. The computational process is realised in an iterative manner, where each subproblem is solved by a reliable numerical method. In the numerical simulations we could observe hysteresis phenomena for the drop deformation and appearance of the drop shapes with ends, close to conical, in the region of high magnetic fields.

\section{Mathematical Model}

We consider a ferrofluid drop of prescribed volume, surrounded by air and located in a uniform magnetic field oriented parallel to the $z$-direction. The mathematical model is formulated in cylindrical coordinates $(r, z, \theta)$ under the assumption of the drop shape to stay axisymmetric as it deforms with a change of the field strength.

Maxwell's equations for a nonconducting media in the stationary case can be written in terms of a magnetic potential $\varphi$ as

$$
-\operatorname{div}(\mu(\xi) \nabla \varphi)=0, \quad \xi \in \Omega ; \quad \mu(\xi)= \begin{cases}1+\chi, & \xi \in \Omega_{F} \\ 1, & \xi \in \Omega \backslash \Omega_{F}\end{cases}
$$

with $\mathbf{H}=-\nabla \varphi$, both inside and outside the drop, where $\mathbf{H}$ is the magnetic field vector. The computational domain $\Omega=$ $\{\xi=(r, z) \mid r \geq 0, z \geq 0\}$ consists of a fluid subdomain $\Omega_{F}$ and a subdomain $\Omega \backslash \Omega_{F}$, filled with air. The magnetic fluid is assumed to be linear magnetisable with a magnetisation law $M(H)=\chi H$, where $\chi$ is the constant magnetic susceptibility. The boundary conditions on an interface $\Gamma$ between two media with different magnetic permeabilities $\mu(\xi)$ must be satisfied

$$
\varphi_{1}=\varphi_{2}, \quad(1+\chi) \frac{\partial \varphi_{1}}{\partial n}=\frac{\partial \varphi_{2}}{\partial n} \quad \text { on } \Gamma,
$$

where the subscripts 1 and 2 denote variables in the fluid and the air domains, respectively. We apply the condition for the magnetic potential to be uniform at the axis $O r$ and axially symmetric at the axis $O z$. We set the condition at infinity $\varphi_{2}=H_{0} z$ such that far from the drop the magnetic field approaches a vertically directed uniform field with the intensity $H_{0}$.

We describe the equilibrium shape of the ferrofluid drop by the parametric functions $r=r(s), z=z(s)$, where $s$ denotes the arc length of the equilibrium line $\Gamma$. The magnetically augmented Young-Laplace equation [4] can be formulated as in [3]

$$
r^{\prime \prime}=-z^{\prime} F, \quad z^{\prime \prime}=r^{\prime} F, \quad 0<s<\ell ; \quad F=-\frac{\mu_{0}}{2 \sigma}\left[\chi H^{2}+\chi^{2} H_{n}^{2}\right]-\frac{z^{\prime}}{r}+C,
$$

where $\ell$ is the unknown arc length of the free surface meridian. Here, $\mu_{0}$ denotes the magnetic permeability, $\sigma$ the surface tension coefficient, $H$ the magnetic field intensity, $H_{n}$ the normal component of the field vector and $C$ is an unknown constant.

\footnotetext{
* Corresponding author: e-mail: olga.lavrova@mathematik.uni-magdeburg.de, Phone: +49391 67 12633, Fax: +4939167 18073.

** e-mail: gunar.matthies@ruhr-uni-bochum.de, Phone: +4923432 23244, Fax: +49234 3214356.

*** e-mail: polevikov@bsu.by, Phone: +375 1720955 32, Fax: +375 172265548.

† e-mail: lutz.tobiska@mathematik.uni-magdeburg.de, Phone: +4939167 18650, Fax: +4939167 18073.
} 


\section{Numerical Solution Strategy}

The numerical solution of the magnetostatic problem (1)-(2) for a fixed free surface shape $\Gamma$ is realised through the coupling of the finite element method inside the fluid domain $\Omega_{F}$ and the boundary element method outside it. A direct boundary element method is based on the Green's representation formula [5]

$$
c\left(\xi^{0}\right) \varphi\left(\xi^{0}\right)-\int_{\Gamma} \varphi(\xi) \frac{\partial \varphi_{a x}^{*}}{\partial n}\left(\xi^{0}, \xi\right) r d \Gamma+\int_{\Gamma} \frac{\partial \varphi(\xi)}{\partial n} \varphi_{a x}^{*}\left(\xi^{0}, \xi\right) r d \Gamma=H_{0} z^{0} \quad \text { for } \xi^{0} \in \Omega \backslash \Omega_{F},
$$

where $c\left(\xi^{0}\right)$ is a geometric constant, $\xi^{0}=\left(r^{0}, z^{0}\right), \varphi_{a x}^{*}\left(\xi^{0}, \xi\right)$ the axisymmetric fundamental solution [5], $n$ the outward unit normal vector to $\Omega_{F}$. We approximate the boundary $\Gamma$ by a parametric cubic spline. As a method for discretising of the integral equation (4) we use a point collocation method in the space of piecewise constant functions [2]. In the weak formulation for the magnetostatic equation (1) inside $\Omega_{F}$ we consider the potential $\varphi$ in $\bar{\Omega}_{F}$ and $\partial \varphi / \partial n$ on $\Gamma$ as unknown functions. We use a standard Galerkin method to discretise the weak formulation with linear finite elements on a triangular grid. Each of the two discrete systems has more unknowns than equations and has to be completed by the equations corresponding the continuity requirements (2). The resulting system is solved by the Gaussian elimination method with pivoting for every fixed surface $\Gamma$.

The numerical solution of the free surface equations (3) for the given field distribution near the boundary $\Gamma$ is realised by a finite difference method of second order on nonuniform grids [3]. An a-priori adaptive method is used to construct the grid on the boundary $\Gamma$, which is dense near the peak of the drop [3]. To solve the corresponding nonlinear difference problem we consider a two-layer iteration scheme [3].

The whole computational process is organised in an iterative manner. The initial boundary $\Gamma_{0}$ is defined as a meridian of a spherical drop with the radius $R_{0}$. At first we solve the magnetostatic problem (1)-(2) for a fixed boundary $\Gamma$ (initial one or last calculated shape). Then we define a new boundary position as a solution of the free surface equations (3) for the new calculated field. The iterative process continues unless the change of the drop shape is smaller than a prescribed threshold.

\section{Results}

According to the theoretical and experimental results in [1] the drop deformation displays hysteresis as a function of the applied field for $\chi>20$. The numerical results (Fig.1. left, circular markers) show quantitative agreement with the theoretical ones (Fig.1. left, solid line) for the lower branch of the solution and only qualitative for the upper branch. The quantitative difference seems to be connected with the assumption of the theoretical model [1] for a drop to be a spheroid for any applied field. Shapes with pointed ends were observed experimentally in [1] and numerically (Fig.1. right) for high field strength.
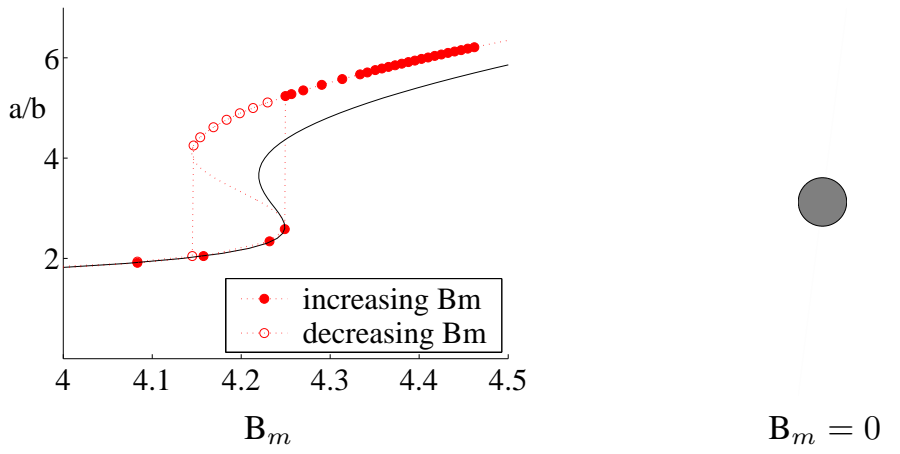

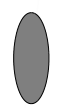

4.249

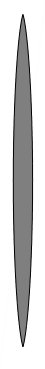

4.25

Fig. 1 The dependence of the drop elongation upon the magnetic field: $\chi=21, B_{m}=\mu_{0} R_{0} H_{0}^{2} /(2 \sigma), a$ and $b$ are lengths of the drop in $z$ - and $r$-directions, respectively. The solid line is a theoretical dependence, the circular markers are the numerical results. On the right side numerically calculated shapes for different $B_{m}$ are presented. $B_{m}=4.249$ is the first turning point, where an abrupt elongation of the drop is observed.

Acknowledgements The authors would like to thank the State Sachsen-Anhalt for supporting the research.

\section{References}

[1] J. C. Bacri, D. Salin, J. Phys. Lett. 43, 649-654 (1982).

[2] W. Hackbusch, Integral equations: theory and numerical treatment, (Birkhauser, Basel, 1995).

[3] V. K. Polevikov, Comput. Methods in Applied Math. Vol. 4, No. 1, 66-93 (2004).

[4] R. E. Rosensweig, Ferrohydrodynamics (Dover Pubns, New York, 1998).

[5] L. C. Wrobel, The boundary element method. Applications in thermo-fluids and acoustics, (Wiley, 2002). 